\title{
What Should Oral Health Clinicians Tell Their Patients About Oral HPV? A Brief Review
}

\author{
Juliet Dang* \\ Oral Health Sciences Alumni, University of Washington, USA \\ Rece||ved: October 31, 2017; Published: November 08, 2017 \\ *Corllesponding author: Juliet Dang, PhD, MS, RDH, Oral Health Sciences, University of Washington, Box 357475, 1959 NE Pacific St., Seattle, WA, 98195 , \\ USA.
}

Abbreviations: STIs: Sexually Transmitted Infections; CDC: Centers for Disease Control and Prevention; OCC: Oral Cavity Cancer; OPC: Oropharyngeal Cancer

\section{Introduction}

In the United States, it is estimated that there are at least 20 million new cases of sexually transmitted infections (STIs) each year, with a prevalence of 110 million people infected, and costing the country 16 billion dollars. The Centers for Disease Control and Prevention (CDC) estimates just over 14 million new cases of HPV infection each year in the US, where half consist of young people between the ages of 15-24 [1]. Approximately 90\% of HPV infections will clear within a few years without any symptoms. However, for the infections that do persist, serious health risks may be the outcome.

\section{HPV Natural History}

We actually are still in the infancy stages regarding the natural history of HPV. However, the information we do have on cervical cancer guides the model for oral cavity cancer (OCC) and oropharyngeal cancer (OPC). HPVs are categorized into low-risk (wart-causing) and high-risk (cancer-causing) types. Globally, HPVs type 16 and 18 cause approximately $70 \%$ of cervical cancers [2]. The other two most common HPV types are 6 and 11, which predominantly cause genital warts. The tissues within the oropharynx are comparable to the tissues of the cervix, thus it is not surprising that we see a similar paradigm occurring between the two regions regarding HPV infection. For example, HPV can be found in precancerous lesions of the oral cavity, which is the same case in cervical cancer [3].

\section{HPV Risk Factors}

Internationally, over time, there have been changes in sexual behaviors with the age of sexual interaction beginning much younger; along with the increasing number of sexual partners one has [4-6]. As well, sexual practices have changed with oral sex being performed more by men and women within the 30-49 year age range compared with older adults [5,6]. As such, it is not surprising that HPV infection of the oral cavity is strongly associated with sexual behavior, but what is still perplexing is that it is twice as predominant in males as in females [7]. A multicenter study observed that the frequency of HPV detection in oral cancer biopsy specimens was higher among individuals who reported having more than one sexual partner or having oral sexual contact [8]. Furthermore, an increased risk of oral cancer has been reported both in women with cervical cancer and in spouses of women with cervical cancer $[8,9]$. These results may collectively suggest a common mode of HPV transmission between the oral and genital area, reinforcing the possibility of sexual transmission of the virus in the oral cavity. Potential reservoirs for the virus are proposed to be the tonsillar crypts $[10,11]$ as well as periodontal pockets [12]. Also, an association between long-standing periodontitis and the risk of tongue cancers has been demonstrated [13]. A recent pilot study observed the oral health status of 223 patients and HPV infection [14]. Their results demonstrated a trend between oral HPV 16 infection and poor clinical oral health status. Thus, it is imperative that oral health practitioners continue to educate their patients on the connections between oral health status and acquiring other diseases.

\section{HPV Prevention}

Abstinence of any sexual behavior, including kissing, would be the most obvious form of prevention, but that is not likely to occur. The Advisory Committee on Immunization Practices (ACIP) recommends the initiation of routine HPV vaccines beginning at the age of 11 or 12 currently there are three different types of HPV vaccines on the market. Gardasil has two kinds available: the 
quadrivalent vaccine, which protects against types $6,11,16$, and 18; and the nonavalent vaccine, which protects against the same types as the quadrivalent vaccine plus five more types $(31,33,45$, 52, and 58). Cervarix has a bivalent vaccine for HPV types 16 and 18. The quadrivalent vaccine was introduced in 2006 and approved for males in 2009. When we look at data extracted between 20032006 and 2009-2012 we see evidence of vaccine efficacy in HPV prevention within women (Figure 1) [15]. For all age groups except for females 25-29, there was a decrease in the prevalence of HPV between the two time periods.

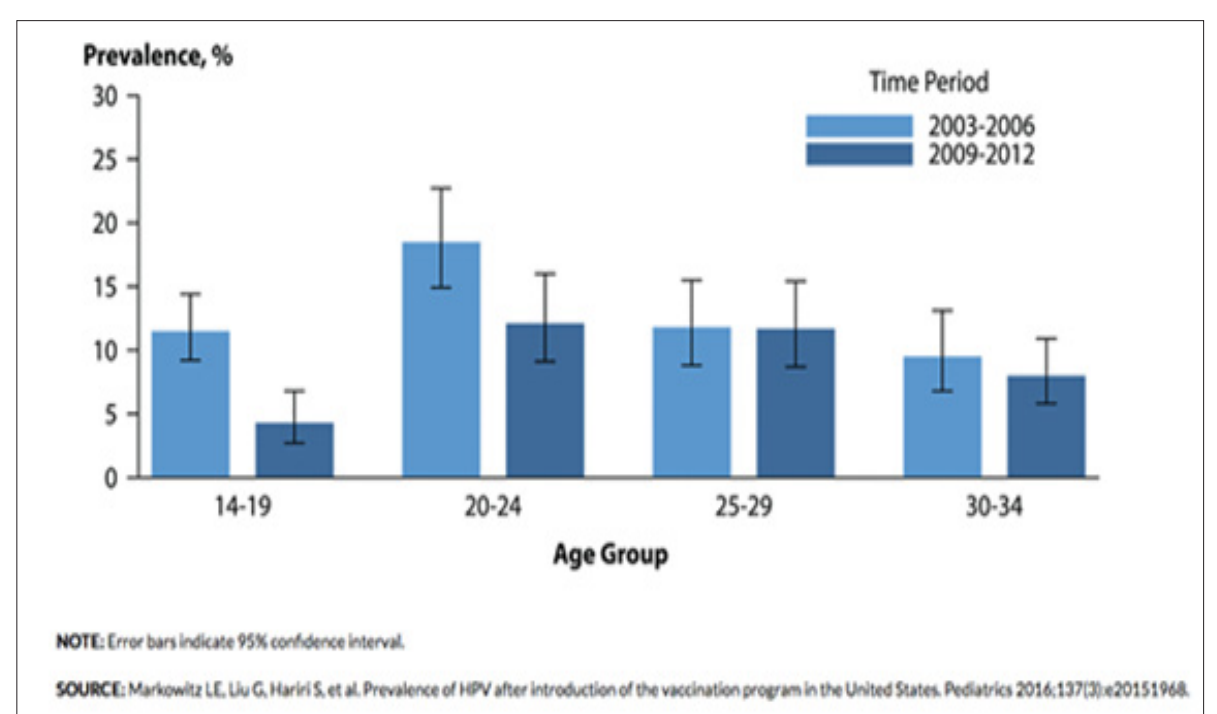

Figure 1: Human Papillomavirus - Cervicovaginal Prevalence of Types 6, 11, 16 and 18 Among Women Aged 14-34 Years by Age Group and Time Period, National Health and Nutrition Examination Survey, 2003-2006 and 2009-2012.

Herrero [16] provided evidence that HPV vaccines used to prevent cervical cancer can be effective in preventing infection of oral HPVs. In a randomized clinical trial in Costa Rica, 7,466 women between the ages of 18-25 years were given the bivalent vaccine for hepatitis A as a control. During the last visit of the blinded 4-year study, 5,840 subjects gave oral specimens in order to evaluate vaccine efficacy (VE) against oral infections. The results demonstrated a VE of $93.3 \%$. The authors theorized that if protection was seen in females the same should occur in males, and that this could be a primary preventative measure for HPV-related oral cancers.

\section{HPV Detection}

Regarding oral HPV detection, there currently are only two companies that provide oral HPV testing. Oral DNA Labs provides their service to only patients that visit their oral health practitioner. SelfCollect.com also provides oral HPV testing that is available to the public without having to visit the dental office. Keiko DNA Labs, a Seattle-based start-up, hopes to provide a more accurate diagnostic test using Real-Time PCR. All sampling methods from each company are non-invasive. HPV-associated OCC/OPC According to the Oral Cancer Foundation, there will be approximately 48,000 new cases of OCC/OPC annually, with a rate increase of $>50 \%$ mortality rate each year globally $[17,18]$. Even with some improvements in scientific efforts and screening, the mortality rate of OCC remains high and the 5-year disease-free survival rate remains quite poor [19].

While HPV-associated OCCs are present, it is the HPV-associated OPCs that are more of a concern as studies have demonstrated HPV association from 30 to $>90 \%$ in OPC cases. Variation between studies results from a variety of factors such as sampling technique (ie. swab, brush, and oral rinse), method of detection (ie. PCR, real-time PCR), and differences in type of molecules being detected (ie. DNA, RNA, p16). The difficult aspect of HPV-associated cancer diagnosing is that transformation of HPV-infected cells to malignant lesion can take many years with little to no symptoms. Treatment of HPVassociated cancers usually consists of excision of tissues infected with malignancy along with chemotherapy or radiation therapy depending on the case.

\section{Closing Remarks}

Oral health practitioners have a plethora of items to discuss with their patients regarding basic oral hygiene care. However, HPV is definitely becoming a topic of conversation while visiting the dental office. Thus, when it comes to educating about oral HPV, take away items from this review are:
a) HPV is the \#1 STI in the US
b) $90 \%$ of HPV infections clear within a few years
c) HPV vaccination is highly recommended for all pre-teens, and may be effective for HPV prevention in adults as well

d) Alcohol consumption, tobacco and marijuana usage, poor oral health, and sexual behaviors can increase risk of HPV infection

e) Oral HPV testing is available

\section{References}

1. (2013) CDC Fact Sheet - Incidence Prevalence, and Cost of Sexually Transmitted Infections in the United States $\mathrm{p} 4$. 
2. Clifford GM, Smith JS, Plummer M, Munoz N, Franceschi S (2003) Human papillomavirus types in invasive cervical cancer worldwide: a metaanalysis. Br J Cancer 88(1): 63-73.

3. Jayaprakash V, Reid M, Hatton E, Merzianu M, Rigual N, et al. (2011) Human papillomavirus types 16 and 18 in epithelial dysplasia of oral cavity and oropharynx: a meta-analysis, 1985-2010. Oral oncology 47(11): 1048-1054.

4. Turner CF, Danella RD, Rogers SM (1995) Sexual behavior in the United States 1930-1990: trends and methodological problems. Sexually transmitted diseases 22(3): 173-190.

5. Herbenick D, Reece M, Schick V (2010) Sexual behavior in the United States: results from a national probability sample of men and women ages 14-94. The journal of sexual medicine 7(5): 255-265.

6. Bajos N, Bozon M, Beltzer N, Laborde C, Andro A, et al. (2010) Changes in sexual behaviours: from secular trends to public health policies. AIDS 24(8): 1185-1191.

7. Gillison ML, Castellsague X, Chaturvedi A, Marc T, Goodman, et al. (2013) Comparative epidemiology of HPV infection and associated cancers of the head and neck and cervix. International journal of cancer.

8. Herrero R, Castellsague X, Pawlita M (2003) Human papillomavirus and oral cancer: the International Agency for Research on Cancer multicenter study. Journal of the National Cancer Institute 95(23): 1772-1783.

9. Rintala M, Grenman S, Puranen M, Syrjanen S (2006) Natural history of oral papillomavirus infections in spouses: a prospective Finnish HPV Family Study. Journal of clinical virology 35(1): 89-94.

10. Hammarstedt L, Lindquist D, Dahlstrand H (2006) Human papillomavirus as a risk factor for the increase in incidence of tonsillar cancer. International journal of cancer. Journal international du cancer 119(11): 2620-2623.
11. Syrjanen S (2004) HPV infections and tonsillar carcinoma. Journal of clinical pathology 57(5): 449-455.

12. Hormia M, Willberg J, Ruokonen H, Syrjanen S (2005) Marginal periodontium as a potential reservoir of human papillomavirus in oral mucosa. Journal of periodontology 76(3): 358-363.

13. Tezal M, Sullivan MA, Reid ME, Marshall JR, Hyland A, et al. (2007) Chronic periodontitis and the risk of tongue cancer. Archives of otolaryngology head \& neck surgery 133(5): 450-454.

14. Sun CX, Bennett N, Tran P, Tang KD, Lim Y, et al. (2017) A Pilot Study into the Association between Oral Health Status and Human Papillomavirus-16 Infection. Diagnostics (Basel)7(1): 11.

15. Markowitz LE, Liu G, Hariri S (2016) Prevalence of HPV After Introduction of the Vaccination Program in the United States. Pediatrics 137(3): 20151968.

16. Herrero R, Quint W, Hildesheim A, Gonzalez P, Struijk L, et al. (2013) Reduced prevalence of oral human papillomavirus (HPV) 4 years after bivalent HPV vaccination in a randomized clinical trial in Costa Rica. PloS one 8(7): 68329.

17. Parkin DM, Bray F, Ferlay J, Pisani P (2005) Global cancer statistics, 2002. CA: a cancer journal for clinicians 55(2): 74-108.

18. Syrjanen S (2005) Human papillomavirus (HPV) in head and neck cancer. Journal of clinical virology 32(1): 59-66.

19. Pannone G, Rodolico V, Santoro A, Lo Muzio L, Franco R, et al. (2012) Evaluation of a combined triple method to detect causative HPV in oral and oropharyngeal squamous cell carcinomas: p16 Immunohistochemistry, Consensus PCR HPV-DNA, and In Situ Hybridization. Infectious agents and cancer 7: 4

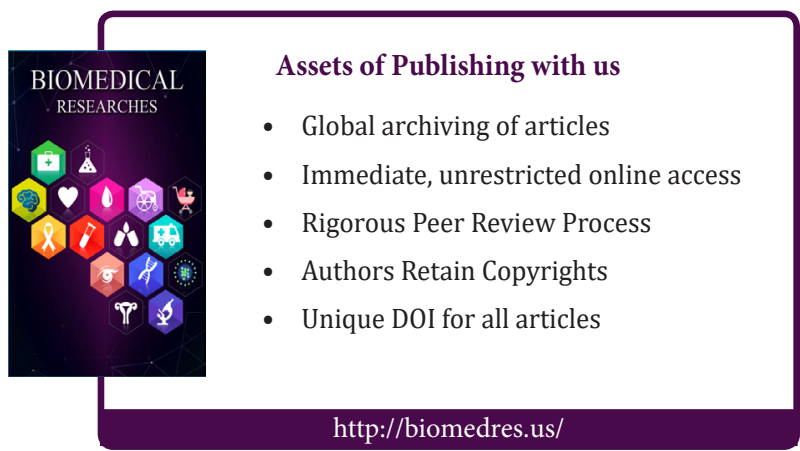

